\title{
QUEEN'S
UNIVERSITY
BELFAST
}

\section{Investigations of ultrafast charge dynamics in laser-irradiated targets by a self probing technique employing laser driven protons}

Ahmed, H., Kar, S., Cantono, G., Nersisyan, G., Brauckmann, S., Doria, D., Gwynne, D., Macchi, A., Naughton, K., Willi, O., Lewis, C. L. S., \& Borghesi, M. (2016). Investigations of ultrafast charge dynamics in laser-irradiated targets by a self probing technique employing laser driven protons. Nuclear Instruments and Methods in Physics Research Section A: Accelerators, Spectrometers, Detectors and Associated Equipment, 829, $172-175$. https://doi.org/10.1016/j.nima.2016.04.078

Published in:

Nuclear Instruments and Methods in Physics Research Section A: Accelerators, Spectrometers, Detectors and Associated Equipment

Document Version:

Peer reviewed version

Queen's University Belfast - Research Portal:

Link to publication record in Queen's University Belfast Research Portal

\section{Publisher rights}

(C) 2016 Elsevier B.V.

This manuscript version is made available under the CC-BY-NC-ND 4.0 licensehttp://creativecommons.org/licenses/by-nc-nd/4.0/,which permits distribution and reproduction for non-commercial purposes, provided the author and source are cited.

\section{General rights}

Copyright for the publications made accessible via the Queen's University Belfast Research Portal is retained by the author(s) and / or other copyright owners and it is a condition of accessing these publications that users recognise and abide by the legal requirements associated with these rights.

Take down policy

The Research Portal is Queen's institutional repository that provides access to Queen's research output. Every effort has been made to ensure that content in the Research Portal does not infringe any person's rights, or applicable UK laws. If you discover content in the Research Portal that you believe breaches copyright or violates any law, please contact openaccess@qub.ac.uk. 


\title{
Investigations of ultra-fast charge dynamics in laser-irradiated targets by a self proton probing technique
}

\author{
H. Ahmed ${ }^{\mathrm{a}}$, S. Kar ${ }^{\mathrm{a}, *}$, G. Cantono ${ }^{\mathrm{a}, \mathrm{b}}$, G. Nersisyan ${ }^{\mathrm{a}}$, S. Brauckmann ${ }^{\mathrm{c}}$, D. Doria ${ }^{\mathrm{a}}$, D. Gwynne ${ }^{\mathrm{a}}$, A. Macchi ${ }^{\mathrm{b}}$, \\ K. Naughton ${ }^{\mathrm{a}}$, O. Willic, C.L.S. Lewis ${ }^{\mathrm{a}}$, M. Borghesi ${ }^{\mathrm{a}}$ \\ ${ }^{a}$ Centre for Plasma Physics, School of Mathematics and Physics, Queen's University Belfast, BT7 1NN, UK \\ ${ }^{b}$ Department of Physics E. Fermi, Largo B. Pontecorvo 3, 56127 Pisa, Italy \\ ${ }^{c}$ Institut für Laser-und Plasmaphysik, Heinrich-Heine-Universität, Düsseldorf, Germany
}

\begin{abstract}
The divergent and broadband proton beams produced by the target normal sheath acceleration mechanism, provide unique opportunity to probe, in a point-projection imaging scheme, the dynamics of the transient electric and magnetic fields produced during laser-plasma interactions. Commonly such experimental setup entails two intense laser beams, where the interaction produced by one beam is probed with the protons produced by the second beam. Here we studied ultra-fast charge dynamics along a wire connected to laser irradiated target by a 'self' proton probing arrangement - i.e. by connecting the wire to the target generating the probe protons. The experimental data shows that an electromagnetic pulse carrying a large amount of charge is launched along the wire, which travels as a unified pulse of 10s of ps duration with a velocity close to speed of light. The experimental capabilities and the analysis procedure of this specific type of proton probing technique are discussed.
\end{abstract}

Keywords:

\section{Introduction}

Amongst different laser-driven acceleration mechanisms currently under development/optimisation, Target Normal Sheath Acceleration (TNSA) mechanism is the most robust and widely studied process [1]. Although the broad energy spectrum and inherent beam divergence of the TNSA protons poses significant scientific and design challenges towards many of its potential applications (for ex. cancer therapy $[2,3]$, warm dense matter creation $[4,5,6]$, production of neutrons [7]), these properties are well suited to radiographic applications [8]. Where the point like source of the quasilaminar and divergent beams of TNSA protons produces radiographs with high spatial resolution (of the order of $\mu \mathrm{m})$, its broad energy spectrum provides a single shot multi-frame capability with high temporal resolution (of the order of $\mathrm{ps}$ ). The proton probing technique has been extensively used for studying transient electric and magnetic fields associated with intense laser plasma interactions $[9,10,11,12]$. A typical setup for these studies employs two temporally synchronised intense laser

\footnotetext{
${ }^{*}$ Corresponding author.

Email address: s.kar@qub.ac.uk
}
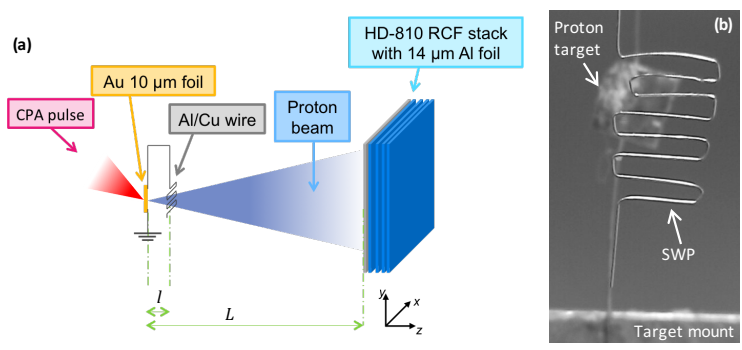

Figure 1: (a) shows a schematic of the experimental setup used for probing the flow of the discharging current in a wire connected to a laser irradiated target in a self proton probing arrangement. (b) Image of the square wave pattern (SWP) target used in the experiment, taken by a 8 bit CCD camera.

pulses. One of the pulses is used to generate the probe protons from a thin metallic foil, while the second pulse with an appropriate target is used to generate the field dynamics, or the physical process, to be investigated.

Here we present investigations of the ultrafast dynamics associated to transient charging of laserirradiated targets, initiated by a intense laser interaction, using a single laser pulse - in an arrangement we refer to as self proton probing (SPP). Transient charging and discharging of laser irradiated targets have 

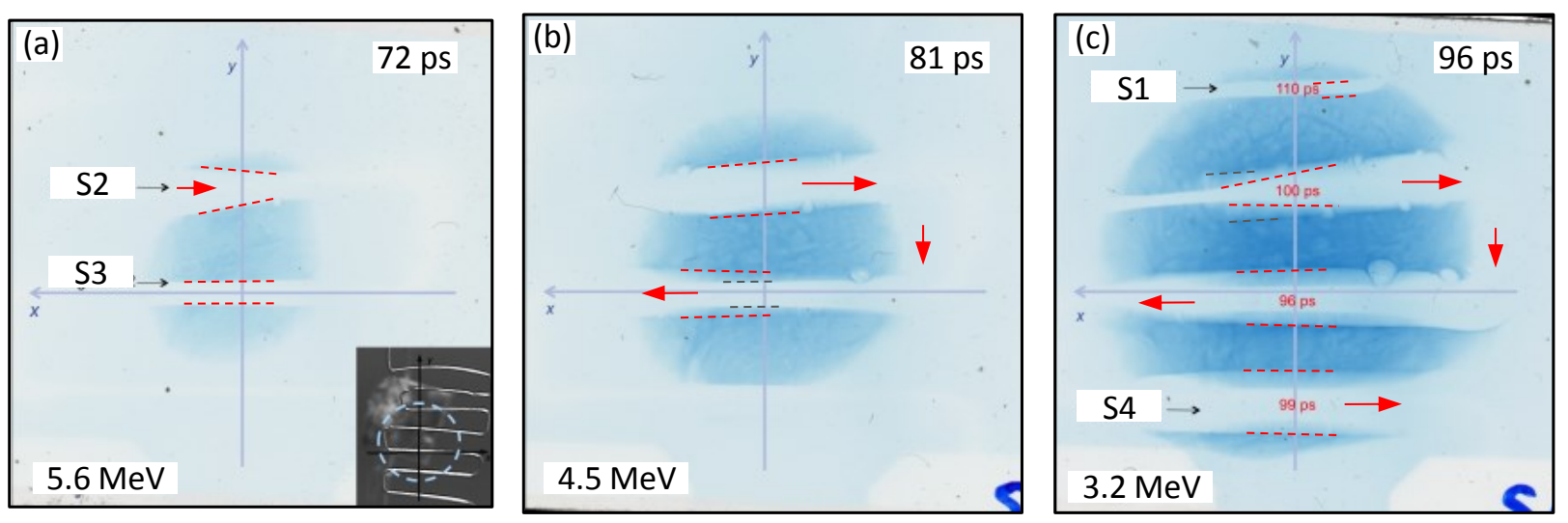

Figure 2: (a), (b) and (c) shows the raw proton images of the charge flow along the SWP at different probing times, obtained in a single shot from different RCF layers in the stack detector, corresponding to Bragg peak proton energy of ( $5.6,4.5 \& 3.2) \pm 0.5) \mathrm{MeV}$ protons (as labelled in the bottom left side of each image). The probing time mentioned at the top-right corner of each image correspond to the time of arrival of respective energy protons at the centre of their field of view on the plane of the SWP. The line segments in the proton images are labelled and the arrows indicate the direction of the charge flow through the SWP, from top to bottom. The red dashed lines provide eye-guide for the width of the proton deflected region around different wire segments for the respective probe proton energies labelled on the image. The insert at the bottom right of (a) shows part of the target image shown in Fig 1(b), with the circle representing the field of view of the probe protons.

been the object of several previous studies employing the standard proton probing technique (using two laser pulses) $[11,12,13,14]$. These studies revealed positive target charge up to MV potential following intense irradiation, due to the escape of relativistic electrons from the laser-irradiated region[11, 12, 15]. The targets were then observed to discharge to ground on timescales of 10 s of ps $[11,14]$. The strong and sudden charge separation caused by the hot electron escape was also seen to lead to the launch of a surface electromagnetic (EM) wave along the target, expanding out from the interaction point at nearly the speed of light $[13,12]$. This surface wave contributes to the target neutralization process by carrying the positive charge away from the interaction region. In this paper we show how an appropriate arrangement allowed us to follow this surface wave along a cm-long wire connected to the laser irradiated target, and to reveal its pulsed nature. We saw the pulse propagating along the wire at a velocity close to the speed of light, while retaining its pulse shape over centimetres of propagation.

\section{Experimental setup}

The experiment was performed using the TARANIS laser at QUB [16], employing the CPA pulse of $\sim 600$ fs pulse duration with energy $\sim 5 \mathrm{~J}$ on target. The short pulse was focused by a $\mathrm{f} / 3$ off axis parabola onto $\sim 10 \mu \mathrm{m}$ thick and a few $\mathrm{mm}^{2}$ gold foil at an intensity $\sim 2 \times 10^{19} \mathrm{~W} / \mathrm{cm}^{2}$. Following this interaction protons are accelerated from the rear surface of the foil via the TNSA process and are used as a charged particle probe for a separate portion of the target. A schematic of the experimental setup is shown in figure 1(a). A stack of multilayer Radiochromic films (RCF) of type HD810 [17] was used as a proton detector. Due to the Bragg peak energy deposition profile of protons in matter, the proton image produced in a given layer of RCF corresponds primarily to a narrow range of proton energy, defined by the position of the RCF layer in the stack.

A particular target design (shown in Fig. 1(a)) was used for studying the charge dynamics far away $(\geq \mathrm{cm})$ from the interaction region. A thin $(\sim 75 \mu \mathrm{m}$ diameter) and several centimeters long Copper wire was connected to the proton-generating gold foil. In order to maximize the length of wire that could be observed within the field of view of the probe beam, the $\mathrm{Cu}$ wire was folded in to a square wave pattern (SWP) in front of and parallel to the interaction foil, as shown in the Fig. 1(b). There were 8 segments in the SWP, as shown in Fig. 1(b), where the length of each horizontal wire segment was $\sim 2.5 \mathrm{~mm}$ and the vertical spacing between two segments was $\sim 600 \mu \mathrm{m}$. The distance between the proton source and the centre of the SWP was $\sim 2.4 \mathrm{~mm}$, whereas the RCF stack detector was placed at $\sim 20 \mathrm{~mm}$ from the proton generating foil, providing a magnification of $\sim 8.3$ in the point-projection arrangement. The length of the $\mathrm{Cu}$ wire from the Au foil to the top of the winding in the SWP was approximately $12 \mathrm{~mm}$, so that a EM wave launched by the interaction and travelling 
along the wire at a velocity close to the speed of light, would be intercepted by the probe proton beam.

\section{Time resolved detection of EM pulse propagation}

Fig. 2 shows the data obtained in three consecutive layers of RCF in the stack detector, which show the propagation of the EM pulse in different segments of the SWP at different probing times. The darkness in the RCF images is proportional to the incident flux of protons of the given energy arriving at the RCF. For an electrically neutral metal wire, a proton radiograph would show a shadow (proton depleted region) of the wire due to multiple-small angle scatterings of the probe protons in the wire. In this case, the width of the shadow on the RCF will be equal to the product of the diameter of the probed wire and the geometrical magnification ( $M=L / l$, where $l$ and $L$ represent the distance from the proton source to the probed wire and the RCF respectively). If the wire is positively charged, the probe protons will experience a strong Coulomb deflection. The width of the proton depleted region on the RCF will in this case be related to the strength of the electric field around the wire and the energy of the probe protons.

As can be seen in Fig. 2(a), the segment S2 appears to be charged to some positive potential, while the next wire segment, S3, remains electrically neutral. The conical shape of the proton deflected region around the segment S2, as highlighted by the red dashed line, indicates the rise of the electric field as positive charge moves along the wire from its left hand side, the side which is connected to the laser irradiated target. At a later probing time, as shown in Fig. 2(b), the segment S3 has become positively charged as evident from the increase of the width of the proton depleted region around S3. This suggests that the charge front associated to the surface wave has flown from S2 to S3 during the time elapsed between the snapshots shown in Fig. 2 (a) and (b). In the following time frame (Fig. 2 (c)), as expected, the charge appears to have entered into the line segment S4. However, it is interesting to observe that the line segment S1 and half of the line segment S2 are back to being electrically neutral at this time. The proton deflected region around S2 takes the form of a reverse conical shape, with the narrower side towards the laser irradiated target. The data therefore are consistent with the propagation of a localised pulse, with a finite temporal width and carrying positive charge. The ${ }_{146}$ propagation of this pulse is consistent with the previous ${ }_{147}$ observation of a surface EM wave generation and prop- 148 agation discussed in [12], and confirms separate obser- ${ }_{149}$ vations reported in [18]. In response to the sudden, pos- 150

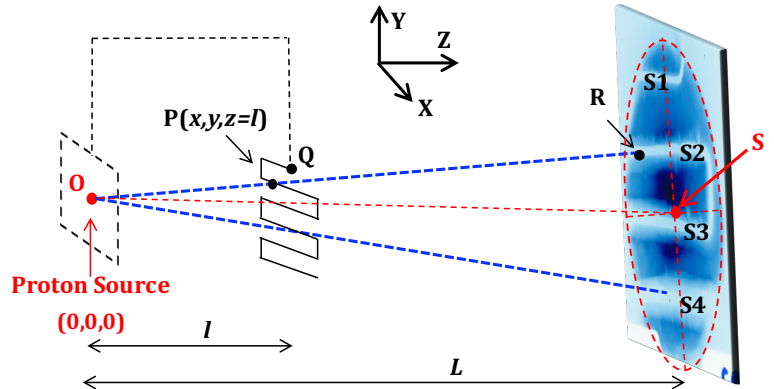

Figure 3: Schematic showing the geometry used for the self proton probing measurement of the neutralising charge flow along the SWP connected to the laser irradiated target. The red dotted line represent the axis of the probe proton beam emitted from a point $\mathrm{P}(0,0,0)$, which is laser interaction point on the flat foil, interacting the plane of the SWP and RCF at distances $l$ and $L$ respectively. $\mathrm{P}(\mathrm{x}, \mathrm{y}, \mathrm{l})$ is a point on one of the line segments of the SWP projected to the point ' $R$ ' on the RCF plane. The line segments of the SWP probed by the protons in this shot is labelled on the RCF image.

itive charge-up of the laser-irradiated area of the target, the propagation of EM pulses away from the interaction regions contributes to lowering the target potential towards neutrality by carrying excess positive charge to remote regions of the target assembly or to ground.

\section{Characterisation of the charge pulse profile}

Quantitative information about the charge pulse temporal profile can be obtained from the data shown in Fig. 2. The energy $\left(E_{\text {proton }}\right)$ with which each of the RCF layers shown in Fig.2 is labelled refers to protons reaching their Bragg peak in the layer, as obtained from SRIM [20] simulations. The probing times labelled at the top right hand corner of the RCF images shown in Fig. 2 correspond to the time of arrival of protons with this energy at the centre of its field of view (see Fig. 3 for a schematic of the geometry involved in our SPP setup). The probing time for different points on the SWP varies within a few ps due to the different path lengths travelled by the probe protons. The absolute probing time at a given location on the plane of the $\operatorname{SWP}(x, y, z=l)$ (see fig. 3) can be calculated by,

$$
t_{\text {proton }}\left(E_{p}, x, y, z=l\right) \simeq \sqrt{\frac{x^{2}+y^{2}+l^{2}}{2 E_{\text {proton }} / m_{p}}}
$$

where $m_{p}$ represents the mass of proton. Assuming the charge pulse is travelling down the wire with a constant speed " $v_{\text {charge" }}$ ", the time of arrival of the charge pulse at the point $\mathrm{P}$ can be written as $t_{\text {charge }}=l_{\text {delay }} / v_{\text {charge }}$, where $l_{\text {delay }}$ is the length of the wire from the proton 


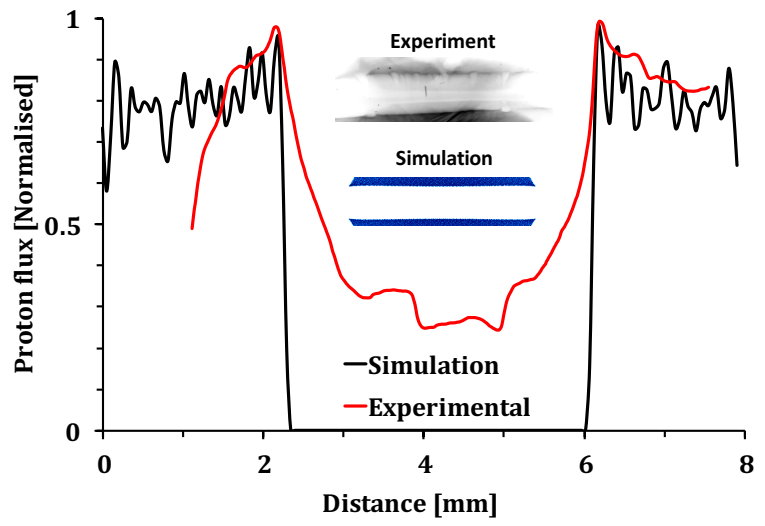

Figure 4: Figure showing the comparison between the experimental and simulated proton flux profiles (as shown in inserts) across a proton depleted region around one of the wire segment shown in Fig. 2.

source to the given point $\mathrm{P}$. Therefore, by measuring the charge density at different points on the SWP at different times, the charge pulse profile can be obtained by ${ }_{205}$ plotting the charge density with respect to the relative ${ }_{206}$ probing time $\left(t=t_{\text {proton }}-t_{\text {charge }}\right)$.

The proton deflection by an electrically charged seg- 208 ment will depend on both the charge density and the 209 probe proton energy. Therefore the 3D particle trac- 210 ing simulations were carried out in order to estimate the 211 charge density from proton deflection. The first step of 212 the analysis was to measure the transverse width of the 213 proton depleted region across the SWP. Although the 214 image obtained in a given RCF layer is produced pri- 215 marily by the protons having their Bragg peak in that 216 layer, the image also contains a fractional contribution 217 from higher energy protons in the probe beam. There- 218 fore each RCF image contains an impression of the im- 219 ages produced by the higher energy protons deeper in 220 the stack [13]. For instance, the gray dotted line around 221 the segment S3 and S2 in Fig. 2(b) and (c), respectively, 222 represent the width of the proton deflection region pro- 223 duced by the higher energy protons, probing the SWP 224 at earlier times, compared to that produced by the pro- 225 tons reaching their bragg peak in the respective layers 226 (marked by the red dotted line). Due to the ultra-fast 227 (close to the speed of the light [12]) propagation of the 228 charge pulse along the wire, these artefact ("ghost") im- 229 pressions becomes an integral part of the proton images 230 produced in the RCF. By considering the dynamics of 231 charging/discharging of a given line segment from the 232 images obtained in different RCF layers, one can iden- 233 tify the actual proton deflection for a given layer from 234 the overlaying ghost impressions. The red dashed lines 235 in Fig. 2 provide eye-guides for the width of the proton 236 deflections around different wire segments in different layers.

In order to find out the local charge density from the width of the proton deflected region, a series of particle tracing simulations using the PTRACE code [21] were carried out. The code was setup to simulate probing of a metallic wire with uniform linear charge density by monoenergetic protons, while using the same geometry and dimensions as per the experimental setup. The simulated proton images were rendered as a two dimensional proton density map at the designated RCF plane in the experiment, i.e. on the plane at a distance $L$ from the proton source. The linear charge density $(\lambda)$ of the wire segment was varied until the width of the proton depleted region matched with the experimental results.

The particle tracing code PTRACE simulates the propagation of the protons from the source through the interaction region and up to the detector. There is a differential equation solver at the core of the PTRACE which computes the trajectory of the particles in presence of electric and magnetic fields. The numerical solver is a Runge-Kutta fourth-order algorithm coupled with an adaptive step-size monitoring routine. The adaptive step-size routine assures that the time steps at which the dynamics are sampled are adequately small so that computational resources are well managed during the simulation for large field strengths. The radial electric field at a given point in space due to the electrically charged wire was calculated by $E(r)=\lambda / 2 \pi \epsilon_{o} r$, where $r$ is the radial distance between the given point and the wire and $\epsilon_{o}$ is the permittivity of vacuum.

The line charge density was varied in the simulation in order to reproduce the experimentally measured width of the proton depleted region around the wire. Fig. 4 shows the comparison between the experimental and a simulated proton flux profiles across a wire segment shown in Fig. 2. By converting the proton deflection to linear charge density, the effect of proton energies on the net deflection produced in different RCF layers was eliminated. This procedure was followed to estimate the local charge density at several points on the segments S2, S3 and S4 wire segments for different probing times.

In order to reconstruct the temporal profile of the charge pulse, the charge densities estimated at different points along the SWP from different RCF layers were plotted as a function of the relative probing time $t=t_{\text {proton }}-t_{\text {charge }}$. The relative probing time accounts for $t_{\text {charge }}$, which depends on the speed of the charge pulse along the wire $\left(v_{\text {charge }}\right)$. Since $l_{\text {delay }}$ for each analysed point on the SWP was measured directly from the target images taken prior to the shot (as shown in 


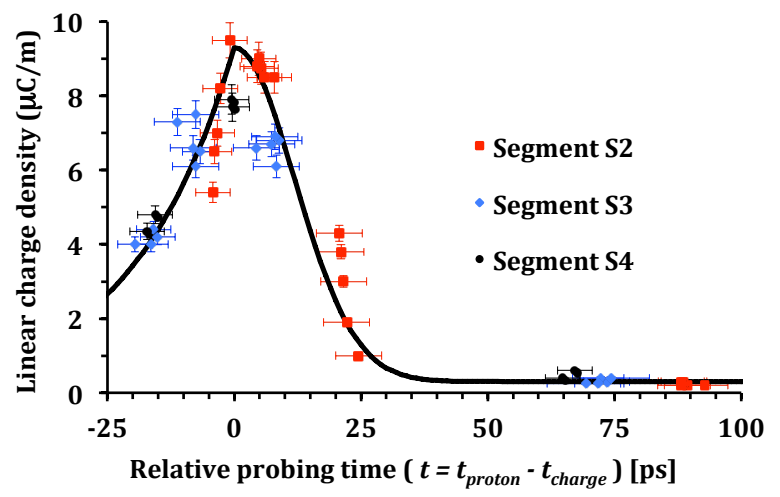

Figure 5: The temporal profile of the charge pulse travelling along 277 the SWP measured by the SPP technique. The graph shows the line charge density measured at different points across different line seg- 278 ments probed by the proton beam with respect to the relative probing 279 time $t=t_{\text {proton }}-t_{\text {charge }}$. The error on the time values is dominated by the width of the Bragg-peak for protons in different RCF layers. The error in the charge density values follows from the uncertainty in 28 measuring the width of the proton deflection from the RCF data.

Fig 1(b)), the charge velocity remains a free parameter in the data set. By varying the value of $v_{\text {charge }}$ in the data points, it was possible obtain a fairly consistent pulse 285 shape centred at $t=0$ as shown in Fig. 5. The agreement between the data points, obtained for different line ${ }_{287}^{286}$ segments at different probing times, was attained for a 288 charge pulse velocity $v_{\text {charge }}=(0.96 \pm 0.04) \mathrm{c}$, where c ${ }^{289}$ is the speed of light in vacuum, which agrees well with ${ }^{290}$ that reported in ref [12]. The error in the velocity mea- ${ }_{292}^{291}$ surement is primarily due to the uncertainty in defining 293 the location of the peak of the charge pulse with respect 294 to the relative probing time. Although a limited num- ${ }^{295}$ ber of temporal snapshots were obtained in this shot, ${ }_{297}^{296}$ one can broadly define the charge pulse as having a full 298 width at half maximum of $\sim 25 \mathrm{ps,} \mathrm{which} \mathrm{is} \mathrm{consistent}{ }^{299}$ with the previous measurements of the neutralization ${ }^{300}$ times of electrically cherged targets $[11,12]$.

\section{Summary and Discussions}

In summary, a slightly different approach to the typ- 307 ical proton radiography technique is discussed. The ${ }^{308}$ technique was used for characterising the charge flow dynamics in a wire connected to a laser irradiated target, ${ }_{311}$ which itself provided the probe protons for the radiog- 312 raphy. The experimental results illustrate that positive charge flows along the wire as a high amplitude pulse of a few tens of ps duration. Furthermore, it is found that the charge can be transported over a long wire (a few $\mathrm{cm}$ ) away from laser interaction region, which, may be important for future developments of schemes useful for controlling and optimising the laser driven proton beams. The electric field associated to such travelling pulse is strong enough to steer $\mathrm{MeV}$ protons, and hence can be used to manipulate the laser driven $\mathrm{MeV}$ proton beams[15]. For instance, allowing the EM pulse to travel along a helical path around the proton beam, the transverse and longitudinal components of the electric field produced inside the helical coil can act simultaneously on a selected bunch (depending on the coil diameter and pitch) of the transiting protons to produce strong focusing and post-acceleration effects [22].

\section{Acknowledgements}

The authors acknowledge funding from EPSRC, [EP/J002550/1-Career Acceleration Fellowship held by S.K., EP/L002221/1, EP/K022415/1, EP/J500094/1 and EP/I029206/1], SBF-TR18 and GRK1203, ECGA284464 and Invest Northern Ireland (POC-329). Authors also acknowledge A. Schiavi for the use of the particle tracing code, PTRACE.

\section{References}

[1] A. Macchi, M. Borghesi, and M. Passoni, Rev. Modern Phys., 85, $751(2013)$

[2] S.V. Bulanov and T.Z. Esirkepov, Phys. Lett. A, 299, 240, (2002); K. Ledingham and W. Galster, The British Journal of Radiology, 80, 855 (2007).

[3] D. Doria et al., AIP Advances,2, 011209 (2012).

[4] M. Tabak et al., Phys. Plasmas, 1, 1626 (1994).

[5] P. Patel et al., Phys. Rev. Lett., 91, 125004 (2003).

[6] M. Koenig et. al, Plasma Phys. Control. Fusion, 47, B441 (2005).

[7] S. Kar et. al, arXiv:1507.04511 [physics.plasm-ph] (2015).

[8] M. Borghesi et al., Phys. Plasmas, 9, 2214, (2002).

[9] S. Kar et al., New J. Phys., 9, 402, (2007).

[10] H. Ahmed et al., Phys. Rev. Lett., 110, 205001 (2013).

[11] M. Borghesi et al., Appl. Phys. Lett., 82, 1529, (2003).

[12] K. Quinn et al., Phys. Rev. Lett., 102, 3 (2009).

[13] K. Quinn et al., Rev. Sci. Instru., 80, 113506 (2009).

[14] K. Quinn et al., Eur. Phys J. D, 55, 293 (2009).

[15] S. Kar et al., Phys. Rev. Lett., 100, 1, (2008).

[16] T. Dzelzainis et al., Laser Particle Beams, 28, 451 (2010).

[17] D Kirby et al., Laser Particle Beams, 29, 231 (2011).

[18] S.Tokita et al., Sci Rep 5, 8268 (2015)

[19] F. Nurnberg et al., Rev. Sci. Instrum. 80033301 (2009).

[20] http://www.srim.org

[21] A. Schiavi, PhD thesis, Imperial College of Science, Technology and Medicine, London,UK, (2003).

[22] S. Kar et al., in press, Nat.Comm (2016). 